\title{
Maximum-Width Empty Square and Rectangular Annulus*
}

\author{
Sang Won Bae Arpita Baral $^{\ddagger} \quad$ Priya Ranjan Sinha Mahapatra ${ }^{\ddagger}$
}

November 16, 2018

\begin{abstract}
An annulus is, informally, a ring-shaped region, often described by two concentric circles. The maximum-width empty annulus problem asks to find an annulus of a certain shape with the maximum possible width that avoids a given set of $n$ points in the plane. This problem can also be interpreted as the problem of finding an optimal location of a ring-shaped obnoxious facility among the input points. In this paper, we study square and rectangular variants of the maximum-width empty anuulus problem, and present first nontrivial algorithms. Specifically, our algorithms run in $O\left(n^{3}\right)$ and $O\left(n^{2} \log n\right)$ time for computing a maximum-width empty axis-parallel square and rectangular annulus, respectively. Both algorithms use only $O(n)$ space.
\end{abstract}

\section{Introduction}

The problem of computing a minimum-size geometric object that encloses an input point set $P$ is one of the central research problems in computational geometry. This type of problem has been extensively studied with direct applications to location of desirable facilities to customers $P$, for a variety of different geometric shapes including circles [18], rectangles [21], and annuli [1$3,5,12,17$.

On the other hand, in some applications, the facility to be built among $P$ is considered obnoxious, that is, every member in $P$ wants to be as far away from it as possible. The problem of locating an obnoxious facility is often interpreted as the problem of finding a maximum-size empty geometric object among $P$. For examples, the center of a largest circle or square that is empty of $P$ corresponds to an optimal location of a point obnoxious facility that maximizes the Euclidean or $L_{\infty}$ distance, respectively, from its closest point in $P$. A largest empty circle or square can be found in optimal $O(n \log n)$ time using the Voronoi diagram [20, and the best known algorithm that computes an empty axis-parallel rectangle of maximum area runs in $O\left(n \log ^{2} n\right)$ time by Aggarwal and Suri [4]. The widest empty corridor problem, in which one wants to find a widest empty strip among $P$ of arbitrary orientation, is another interesting problem in this concept. After Houle and Maciel [14] presented an $O\left(n^{2}\right)$-time algorithm for this problem, a lot of variants and extensions have been addressed, including the widest L-shaped corridor problem [7], and the widest 1-corner corridor problem [9]. Note that these problems

${ }^{*}$ S. W. Bae was supported by Basic Science Research Program through the National Research Foundation of Korea (NRF) funded by the Ministry of Education (2018R1D1A1B07042755). P. R. S. Mahapatra was supported by Research Project through Department of Atomic Energy (NBHM), Goverment of India with Ref. No. 2/48(19)/2014/R\&D-II/1045.

${ }^{\dagger}$ Division of Computer Science and Engineering, Kyonggi University, Suwon, Republic of Korea. Email: swbae@kgu.ac.kr.

${ }^{\ddagger}$ Department of Computer Science and Engineering, University of Kalyani, India. Emails: arpitabaral@gmail.com, priya@klyuniv.ac.in. 
are equivalent to those of finding an optimal location of an obnoxious facility whose shape is of a line, a line segment, or a polygonal chain.

In this paper, along this line of research, we study the maximum-width empty annulus problem. Informally, an annulus is a ring-shaped region, often described by two concentric circles. Thus, the maximum-width empty annulus problem is to find an optimal location of a ring-shaped obnoxious facility among the input points $P$. Specifically, we discuss its square and rectangular variants, and present first nontrivial algorithms. Our algorithms run in $O\left(n^{3}\right)$ and $O\left(n^{2} \log n\right)$ time for computing a maximum-width axis-parallel square and rectangular annulus, respectively, that is empty of a given set $P$ of $n$ points in the plane. Both algorithms use only $O(n)$ space.

There has been a little work on the maximum-width empty annulus problem. Díaz-Báñez et al. 10] first studied the problem for circular annulus, and proposed an $O\left(n^{3} \log n\right)$-time and $O(n)$ space algorithm to solve it. To our best knowledge, there was no known correct algorithm in the literature for the maximum-width empty square or rectangular annulus problem. Mahapatra [16 considered the maximum-width empty rectangular annulus problem and claimed an incorrect $O\left(n^{2}\right)$-time algorithm. There is a missing argument in Observation 2 of [16], which incorrectly claimed that the total number of potential outer rectangles forming an empty rectangular annulus is $n-1$.

Unlike the maximum-width empty annulus problem, the problem of finding a minimum-width annulus that encloses $P$ has recently attained intensive interests from researchers. As a classical one, circular annuli have been studied earlier with applications to the roundness problem 11 , 19, 22], and the currently best known algorithm runs in $O\left(n^{3 / 2+\epsilon}\right)$ time [2, 3. Computing a minimum-width axis-parallel square or rectangular annulus that encloses $n$ points $P$ can be done in $O(n \log n)$ or $O(n)$ time, respectively [1,12]. Mukherjee et al. [17] considered the problem of identifying a rectangular annulus of minimum width that encloses $P$ in arbitrary orientation, and presented an $O\left(n^{2} \log n\right)$-time algorithm. Bae [5] studied a minimum-width square annulus in arbitrary orientation and showed that it can be solved in $O\left(n^{3} \log n\right)$ time.

The rest of the paper is organized as follows: In Section 2, we introduce some definitions and notations, and precisely define our problems. Our algorithms are described in the following sections: Section 3 for computing a maximum-width empty axis-parallel square annulus and Section 4 for computing a maximum-width empty axis-parallel rectangular annulus. Finally, we finish the paper with concluding remarks in Section 5.

\section{Problem Definition and Terminologies}

Throughout the paper, we consider a Cartesian coordinate system of the plane $\mathbb{R}^{2}$ with the $x$ and $y$-axes. For any point $p$ in the plane $\mathbb{R}^{2}$, we denote by $x(p)$ and $y(p)$ its $x$ - and $y$-coordinates. For an axis-parallel rectangle or square, its four sides are naturally identified by top, bottom, left, and right sides, respectively.

For an axis-parallel square, the intersection point of its two diagonals is called its center, and its radius is half its side length. An axis-parallel square annulus is the region between two concentric axis-parallel squares $S$ and $S^{\prime}$, where $S^{\prime} \subseteq S$. We call $S$ and $S^{\prime}$ the outer and inner squares, respectively, of the annulus. The width of a square annulus is defined to be the difference of radii of its outer and inner squares. See Figure 11(left) for an illustration.

An axis-parallel rectangular annulus is the region obtained by subtracting the interior of an axis-parallel rectangle $R^{\prime}$ from another axis-parallel rectangle $R$ such that $R^{\prime} \subseteq R$. We call $R$ and $R^{\prime}$ the outer rectangle and inner rectangle of the annulus, respectively. Consider a rectangular annulus $A$ defined by its outer and inner rectangles, $R$ and $R^{\prime}$. By our definition, note that $R$ and $R^{\prime}$ defining annulus $A$ do not have to be concentric, so that $A$ may not be a symmetric shape. The top-width of $A$ is the vertical distance between the top sides of $R$ and $R^{\prime}$, 

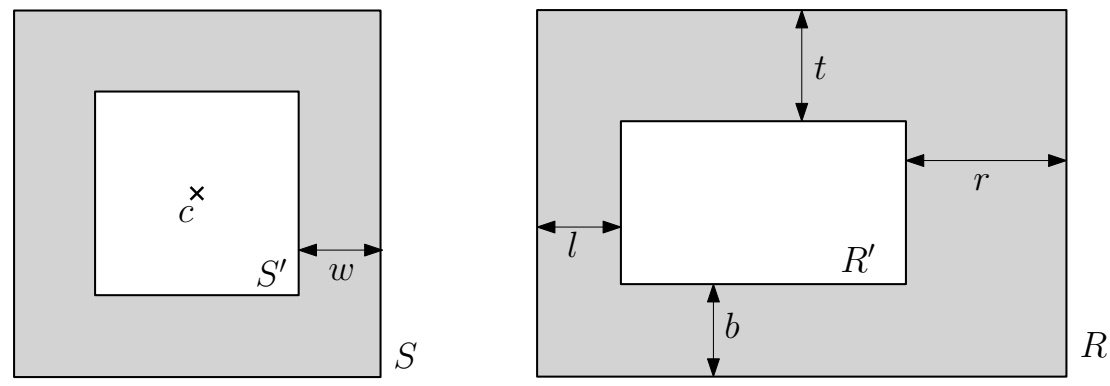

Figure 1: A square annulus of width $w$ with outer and inner squares $S$ and $S^{\prime}$ having a common center $c$ (left) and a rectangular annulus with outer and inner rectangles $R$ and $R^{\prime}$ whose top-, bottom-, left-, right-widths are $t, b, l$, and $r$, respectively (right).

and the bottom-width of $A$ is the vertical distance between their bottom sides. Analogously, the left-width and right-width of $A$ are defined to be the horizontal distance between the left sides of $R$ and $R^{\prime}$ and the right sides of $R$ and $R^{\prime}$, respectively. Then, the width of $A$ is defined to be the minimum of the four values: the top-width, bottom-width, left-width, and right-width of $A$. See Figure 1(right) for an illustration.

In this paper, we only discuss squares, rectangles, square annuli, and rectangular annuli that are axis-parallel. Hence, we shall drop the term "axis-parallel", and any square, rectangle, or annulus we discuss is assumed to be axis-parallel.

Let $P$ be a set of $n$ points in $\mathbb{R}^{2}$. A square or rectangular annulus $A$ is said to be empty of $P$, or just empty when there is no confusion, if the interior of $A$ does not contain any point in $P$. Consider any empty square or rectangular annulus $A$. Then, $A$ induces a partition of $P$ into two subsets $P_{\text {out }}$ and $P_{\text {in }}$ such that $P_{\text {in }}$ is the set of points in $P$ lying in the interior or on the boundary of the inner square or rectangle of $A$, and $P_{\text {out }}=P \backslash P_{\text {in }}$. If both $P_{\text {out }}$ and $P_{\text {in }}$ are nonempty, then we say that $A$ is valid. In this paper, we address the following problems:

MAXWidthEMPTySQUAREANnulus (MaxESA)

Input: A set of points $P$ in $\mathbb{R}^{2}$

Output: A valid empty square annulus $A$ of maximum width

MAXWidThEMPtyRECTANGULARANnulus (MaxERA)

Input: A set of points $P$ in $\mathbb{R}^{2}$

Output: A valid empty rectangular annulus $A$ of maximum width

The constraint that the resulting empty annulus should be valid is essential to make the problem nontrivial; the same constraint has often been considered in the problem of computing empty objects of maximum size $[7,9,10,15]$. Throughout the paper, we are interested only in valid empty annuli, so we shall drop the term "valid" unless stated otherwise.

\section{Maximum-Width Empty Square Annulus}

In this section, we present an algorithm that computes a maximum-width valid empty square annulus for a given set $P$ of $n$ points.

Consider any empty square annulus $A$. Keeping the same partition of $P$ by $A$, one can enlarge the outer square and shrink the inner square so that some points of $P$ lie on the boundary of the outer and inner squares. This process implies the following observation. A side of a rectangle 
or a square is said to be at infinity if it is a translated copy of a line segment by a translation vector at infinity.

Observation 1 There exists a maximum-width empty square annulus such that one side of its inner square contains a point of $P$ and one of the following holds: (i) There are a pair of opposite sides of its outer square, each of which contains a point of $P$, (ii) there are two adjacent sides of its outer square, each of which contains a point of $P$, and the other two sides are at infinity, or (iii) One side of its outer square contains a point of $P$ and the other three sides are at infinity.

Proof. Consider any maximum-width empty square annulus $A$ with outer square $S$, inner square $S^{\prime}$, and center $c$. If there is no point on the boundary of the inner square $S^{\prime}$, then we can shrink it with the same center, since $A$ is valid, so that we can strictly increase its width, a contradiction. So, there must be at least one point lying on the boundary of $S^{\prime}$. Similarly, if $S$ contains no point of $P$ on its boundary, then we can enlarge it with the same center, keeping $S^{\prime}$ the same, so that we can strictly increase its width, a contradiction. So, there must be at least one point $p \in P$ lying on the boundary of $S$.

Without loss of generality, we assume that $p$ lies on the top side of $S$. If the other three sides of $S$, except the top side, contain no point of $P$, then we move the center $c$ in the direction away from $p$, keeping $p$ on the top side of $S$ and the width of $A$ unchanged, until one of the other three sides of $S$ hits a point of $P$. If this enlarging process does not stop, then we end with the three sides of $S$ being at infinity, resulting in case (iii). Otherwise, we now have another side of $S$ containing a point $p^{\prime} \in P$. There are two cases: either it is the bottom side of $S$, which is now in case (i), or is the left or right side of $S$.

In the latter case, we have two adjacent sides of $S$ each of which contains a point of $P$. Without loss of generality, we assume the second side is the left side. Now, we again move the center in the direction away from the top-left corner of $S$, keeping $p$ on the top side of $S, p^{\prime}$ on the left side of $S$, and the width of $A$ unchanged, until one of the bottom and right sides hits a point of $P$. If this enlarging process does not stop, then we end with the two sides of $S$ being at infinity, resulting in case (ii). Otherwise, we now have the third side of $S$ containing a point of $P$, and this falls in case (i).

By Observation 1, we now have three different configurations of empty square annuli to search for. If this is case (iii), then observe that it corresponds to a maximum-width empty horizontal or vertical strip, which also can be reduced to the problem of finding the maximum gap in $\{x(p) \mid p \in P\}$ or in $\{y(p) \mid p \in P\}$. Hence, case (iii) can be handled in $O(n)$ time after sorting $P$.

On the other hand, if this is case (ii), then the resulting square annulus corresponds to a maximum-width empty "axis-parallel" L-shaped corridor. It is known that a maximum-width empty L-shaped corridor over all orientations can be computed in $O\left(n^{3}\right)$ time with $O\left(n^{3}\right)$ space by Cheng [7], while we are seeking only for axis-parallel ones. Here, we give a simple $O\left(n^{2} \log n\right)$ time algorithm for this problem.

Theorem 1 Given $n$ points in the plane, one can compute a widest empty axis-parallel corridor in $O\left(n^{2} \log n\right)$ time using $O(n)$ space.

Proof. Consider the induced grid by points in $P$ obtained by drawing the horizontal and vertical lines through every point in $P$. For each grid point $o$, consider the four quadrants at $o$ obtained by the vertical and horizontal lines through $o$. Consider the first quadrant, or equivalently the top-right quadrant, $Q$. By finding out the point with the smallest $x$-coordinate and the point with the smallest $y$-coordinate among $P \cap Q$, we can identify a candidate L-shape empty corridor with corner $o$. This query can be handled in $O(\log n)$ time by the segment dragging data structure by Chazelle [6] which can be built in $O(n \log n)$ time using $O(n)$ space. 


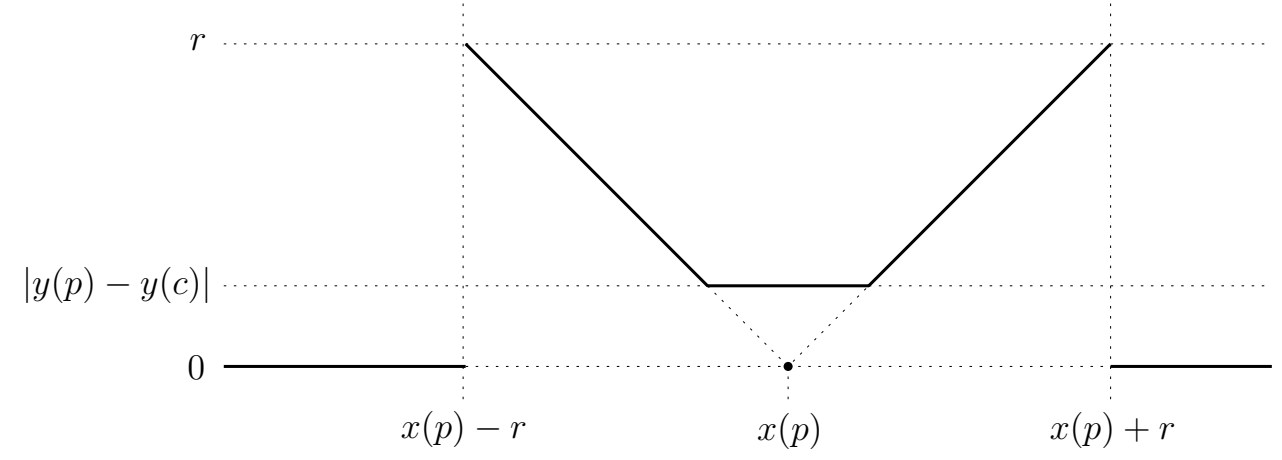

Figure 2: Illustration of the graph of function $f_{p}$ for $p \in P$.

Since there are $O\left(n^{2}\right)$ grid points, we can compute a maximum-width empty axis-parallel corridor in $O\left(n^{2} \log n\right)$ time.

Now, we suppose that the solution falls in case (i) of Observation 1, so that both two opposite sides of its outer square contains a point of $P$. Without loss of generality, we assume that each of the top and bottom sides of the outer square of our target annulus contain a point of $P$. The other case can be handled in a symmetric way.

First, as preprocessing, we sort $P$ in the decreasing order of their $y$-coordinates, so $P=$ $\left\{p_{1}, p_{2}, \ldots, p_{n}\right\}$, where $y\left(p_{1}\right) \geq \cdots \geq y\left(p_{n}\right)$. We also maintain the list of points in $P$ sorted in their $x$-coordinates. Our algorithm runs repeatedly for all pairs of indices $(i, j)$ with $1 \leq i<$ $j-1<n$, and finds a maximum-width empty square annulus such that the top and bottom sides of its outer square contain $p_{i}$ and $p_{j}$, respectively.

From now on, we assume $i$ and $j$ are fixed. Let $P_{i j}:=\left\{p_{i+1}, \ldots, p_{j-1}\right\}, r:=\left(y\left(p_{i}\right)-y\left(p_{j}\right)\right) / 2$, and $\ell$ be the horizontal line with $y$-coordinate $y(\ell)=\left(y\left(p_{i}\right)+y\left(p_{j}\right)\right) / 2$. Provided that $p_{i}$ lies on the top side and $p_{j}$ lies on the bottom side of the outer square, the possible locations of its center is constrained to be on $\ell$. For a possible center $c \in \ell$, let $S(c)$ be the square centered at $c$ with radius $r$. Then, the corresponding inner square $S^{\prime}(c)$ is determined by center $c$ and the farthest point among those points in $P_{i j}$ lying in the interior of $S(c)$. Here, the distance is measured by the $L_{\infty}$ metric. More precisely, the radius of $S^{\prime}(c)$ is exactly $\max _{p \in P_{i j} \cap S(c)}\|p-c\|_{\infty}$, where $\|\cdot\|_{\infty}$ denotes the $L_{\infty}$ norm, and we want to minimize this over the relevant segment $C \subset \ell$ such that $S(c)$ for $c \in C$ contains $p_{i}$ and $p_{j}$ on its top and bottom sides. Note that the length of segment $C \subset \ell$ is exactly $2 r-\left|x\left(p_{i}\right)-x\left(p_{j}\right)\right|$.

For the purpose, we define $f_{p}(c)$ for each $p \in P_{i j}$ and all $c \in \ell$ to be

$$
f_{p}(c)=\left\{\begin{array}{ll}
\|p-c\|_{\infty} & \text { if }\|p-c\|_{\infty}<r \\
0 & \text { otherwise }
\end{array},\right.
$$

and let $F(c):=\max _{p \in P_{i j}} f_{p}(c)$ be their upper envelope. Note that our goal is to minimize the upper envelope $F$ over $C \subset \ell$.

Note that $x(p)-r<x(c)<x(p)+r$, or equivalently, $p \in S(c)$ if and only if $\|p-c\|_{\infty}<r$ for any $p \in P_{i j}$. As also observed in Bae [5], the function $f_{p}$ is piecewise linear with at most three pieces over $c \in \ell$ such that $p \in S(c)$, and the three pieces have slopes $-1,0$, and 1 in this order. Moreover, the height of the part of slope 0 is exactly $|y(p)-y(c)|=|y(p)-y(\ell)|$ and the extensions of the two pieces of slope -1 and 1 always cross at the point of $x$-coordinate $x(p)$ and height 0 . See Figure 2. These properties of $f_{p}$ can be easily verified from the behavior of the $L_{\infty}$ norm. By the above observations, one can explicitly compute $F$ by computing the upper 
envelope of $O(n)$ line segments in $O(n \log n)$ time [13]. Applying this to all possible pairs $(i, j)$ yields an $O\left(n^{3} \log n\right)$ time algorithm.

In the following, we show how to improve this to $O\left(n^{3}\right)$ time by decomposing the function $f_{p}$ into two functions $g_{p}$ and $h_{p}$. For each $p \in P_{i j}$ and $c \in \ell$, define

$$
g_{p}(c)=|x(p)-x(c)| \quad \text { and } \quad h_{p}(c)=|y(p)-y(c)| \quad \text { if }\|p-c\|_{\infty}<r,
$$

and $g_{p}(c)=h_{p}(c)=0$, otherwise. Also, let

$$
G(c):=\max _{p \in P_{i j}} g_{p}(c) \quad \text { and } \quad H(c):=\max _{p \in P_{i j}} h_{p}(c) .
$$

As $\|p-c\|_{\infty}=\max \{|x(p)-x(c)|,|y(p)-y(c)|\}$, it is obvious that $f_{p}(c)=\max \left\{g_{p}(c), h_{p}(c)\right\}$, and hence $F(c)=\max \{G(c), H(c)\}$. We now show that the functions $G$ and $H$ can be explicitly computed in $O(n)$ time.

Lemma 1 The functions $G$ and $H$ can be explicitly computed in $O(n)$ time.

Proof. First recall that we know the list of points in $P$ sorted in their $x$-coordinates in the preprocessing. From this list, we can find out the list of points in $P_{i j}$ sorted in the $x$-coordinates in linear time.

By above discussions, the graph of function $g_{p}$ consists of exactly two line segments, except of the two parts of zero, of slopes -1 and 1 , and the breakpoint lies at $x(p)$. That is, all the functions $g_{p}$ are translates of this V-shaped segments. We first compute the upper envelope of all segments of slope -1 of $g_{p}$. This can be done in $O(n)$ time since we know their sorted order. Analogously, we compute the upper envelope of all segments of slope 1 of $g_{p}$ in $O(n)$ time. Merging these two envelope into the upper envelope $G$ can be done in $O(n)$ time. Thus, we can compute $G$ in $O(n)$ time.

To compute $H$, observe that the graph of function $h_{p}$ consists of two zero parts and a horizontal line segment of length $2 r$. Since every non-zero segment in functions $h_{p}$ for $p \in P_{i j}$ has the same length and we know the sorted order, we can compute their upper envelope $H$ in $O(n)$ time.

Since the function $F$ is the upper envelope of $G$ and $H$, we can compute $F$ in $O(n)$ time using the explicit description of functions $G$ and $H$. Note that the three functions $F, G$, and $H$ are piecewise linear with $O(n)$ breakpoints. Consequently, we can compute $F$ and find a lowest point of $F$ over $C \subset \ell$ in $O(n)$ time, and hence a maximum-width empty square annulus of case (i) can be found in $O\left(n^{3}\right)$ time. Finally, we conclude the following theorem.

Theorem 2 Given $n$ points in the plane, a maximum-width empty square annulus can be computed in $O\left(n^{3}\right)$ time using $O(n)$ space.

Proof. Recall Observation 1 stating that there are three cases of a possible maximum-width empty square annulus. In the overall algorithm, we check all the three possibilities as follows: For case (i), we run the above algorithm in $O\left(n^{3}\right)$ time. For case (ii), we compute a maximumwidth empty axis-parallel L-shaped corridor in $O\left(n^{2} \log n\right)$ time by Theorem 1. For case (iii), we compute a maximum-width empty axis-parallel strip in $O(n)$ time. Thus, the correctness directly follows from Observation 1 . All these routines use $O(n)$ space.

\section{Maximum-Width Empty Rectangular Annulus}

In this section, we present an algorithm computing a maximum-width empty rectangular annulus. First we give several basic observations on maximum-width empty rectangular annuli. 

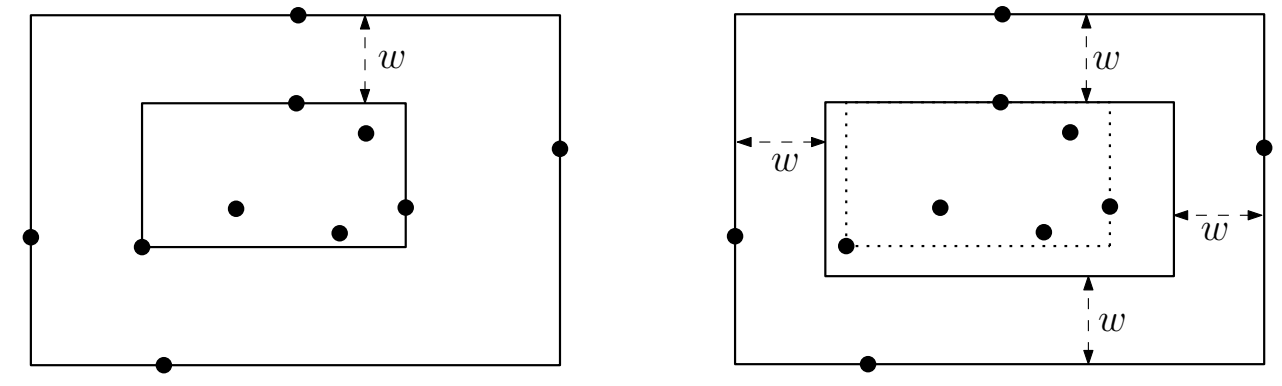

Figure 3: Empty rectangular annuli of maximum width $w$ : (left) Each side of the inner and outer rectangles contains a point. (right) A maximum-width empty rectangular annulus that is uniform and also top-anchored.

\subsection{Configurations of empty rectangular annuli}

Consider any empty rectangular annulus $A$ and the partition of $P$ induced by $A$. As done for square annuli before, one can enlarge the outer rectangle of $A$ and shrink its inner rectangle, while keeping the partition of $P$ and not decreasing the width of $A$. This results in the following observation.

Observation 2 There exists a maximum-width empty rectangular annulus such that each side of its outer rectangle either contains a point of $P$ or lies at infinity, and every side of its inner rectangle contains a point of $P$.

Proof. Let $A$ be any empty rectangular annulus with outer rectangle $R_{\text {out }}$ and inner rectangle $R_{\text {in }}$. We first enlarge $R_{\text {out }}$ to obtain a new outer rectangle $R_{\text {out }}^{\prime}$ as follows: If the top side of $R_{\text {out }}$ contains no point of $P$, then we slide it upwards until it hits a point of $P$. If this process stops at some point, then the top side contains a point of $P$; otherwise, it is now at infinity. Afterwards, we perform the same process for the other three sides, one by one. Hence, each side of the resulting rectangle $R_{\text {out }}^{\prime}$ either contains a point of $P$ or lies at infinity.

Next, we shrink $R_{i n}$ by sliding each side of $R_{i n}$ inwards until it hits a point of $P$, and let $R_{i n}^{\prime}$ be the resulting rectangle. Since $A$ is assumed to be valid, there is at least one point of $P$ in the interior or on the boundary of $R_{i n}$, which implies that every side of $R_{i n}^{\prime}$ contains a point of $P$.

Now, consider a new rectangular annulus $A^{\prime}$ defined by its outer rectangle $R_{\text {out }}^{\prime}$ and inner rectangle $R_{i n}^{\prime}$. By the above processes, it is obvious that $A^{\prime}$ is also empty and the width of $A^{\prime}$ is not smaller than that of $A$. Moreover, $A^{\prime}$ satisfies the condition described in the statement. By our construction, we show that there always exists such an snnulus $A^{\prime}$ for any empty rectangular anuulus, so the observation follows.

In Observation 2, note that each side of a rectangle is considered to include its endpoints. Thus, a point $p \in P$ can be contained in two adjacent sides of a rectangle if $p$ is located at a corner. See Figure 3 (left).

A rectangular annulus $A$ is said to be width-uniform, or simply uniform, if its top-width, bottom-width, left-width, and right-width are all equal to its width. In the following observation, we show that we can focus only on uniform rectangular annuli to solve our problem.

Observation 3 There exists a maximum-width empty rectangular annulus $A$ that is uniform such that the following property holds: each side of its outer rectangle either contains a point of $P$ or lies at infinity, and at least one side of its inner rectangle contains a point of $P$.

Proof. Let $A$ be a maximum-width empty rectangular annulus with the property described in 
Observation 2, Let $R_{\text {out }}$ and $R_{\text {in }}$ be the outer and inner rectangles of $A$, and $w$ be the width of $A$.

Without loss of generality, we assume that $w$ is equal to the top-width of $A$, that is, the top-width is the smallest among the other three. We then enlarge the inner rectangle $R_{i n}$ to have a new inner rectangle $R_{i n}^{\prime}$ by sliding the left side to the left, the right side to the right, and the bottom side downwards so that the resulting annulus $A^{\prime}$ formed by $R_{\text {out }}$ and $R_{\text {in }}^{\prime}$ is uniform and the width of $A^{\prime}$ is equal to $w$, the width of $A$. See Figure 3 for an illustration. Since $R_{i n} \subseteq R_{i n}^{\prime}$ and thus $A^{\prime} \subseteq A$, we conclude that $A^{\prime}$ is also empty of $P$. The proof is done by also observing that every side of $R_{\text {out }}$ contains at least one point of $P$ and the top side of $R_{\text {in }}^{\prime}$ contains at least one point of $P$ by Observation 2 .

See Figure 3(right) for an illustration of Observation 3 . This observation suggests a specific configuration of annuli for us to solve the problem. First of all, we do not have to consider non-uniform annuli. Moreover, candidate outer rectangles are defined by at most four points in $P$. If we fix an outer rectangle $R$, then the inner rectangle that maximizes the width is also determined by searching points in $P \cap R$. This already yields an $O\left(n^{5}\right)$-time algorithm for our problem.

Let $A$ be an empty rectangular annulus satisfying the condition described in Observation 2 , We call A top-anchored (or, bottom-anchored, left-anchored, right-anchored) if both the top sides (or, bottom sides, left sides, right sides, resp.) of the outer and inner rectangles of $A$ contain a point of $P$. For example, Figure 3(right) shows an empty top-anchored rectangular annulus.

Observation 4 There exists a maximum-width empty rectangular annulus $A$ that satisfies the condition described in Observation 3 and is either top-anchored, bottom-anchored, left-anchored, or right-anchored.

Proof. Let $A$ be a maximum-width empty rectangular annulus that satisfies the condition described in Observation 3. Note that $A$ is guaranteed to be uniform. Now, suppose that $A$ is neither top-anchored, bottom-anchored, left-anchored, nor right-anchored. By Observation 3, at least one side of the inner rectangle of $A$ contains a point of $P$. Without loss of generality, assume that the top side of the inner rectangle contains a point of $P$. Since $A$ is not top-anchored, the top side of the outer rectangle contains no point of $P$ and is thus at infinity. This implies that its top-width is unbounded. Since $A$ is uniform, this should be the case for the other three bottom-width, left-width, and right-width. This means that all sides of the outer rectangle of $A$ lie at infinity, and that no point of $P$ lies on the boundary or outside of the outer rectangle. This contradicts to the assumption that $A$ is valid.

Our algorithm will find an empty anchored and uniform rectangular annulus of maximum width, which is the correct answer to our problem by Observation 4 . In the following, we assume without loss of generality that there exists a maximum-width empty annulus that is uniform and top-anchored, and describe our algorithm for this case. The other three cases can be handled analogously.

Let $P=\left\{p_{1}, p_{2}, \ldots, p_{n}\right\}$ be the given set of points, sorted in the descending order of their $y$ coordinates, that is, $y\left(p_{1}\right) \geq y\left(p_{2}\right) \geq \cdots \geq y\left(p_{n}\right)$. Consider any empty top-anchored rectangular annulus $A$ that satisfies the condition of Observation 3 . Let $p_{i} \in P$ be the point lying on the top side of the outer rectangle of $A$. By Observation 3, either the bottom side of the outer rectangle is at infinity or there is another point $p_{j} \in P$ for $i<j \leq n$ on it. If the bottom side is at infinity, then we say that a point $p_{\infty}$ at infinity in the $(-y)$-direction lies on the bottom side. Thus, in either case, there is $p_{j}$ on the bottom side of the outer rectangle for $i<j \leq n$ or $j=\infty$.

Since $A$ is top-anchored, there is a third point $p_{k} \in P$ on the top side of the inner rectangle of $A$. Observe that the width of $A$ is determined by the $y$-difference of $p_{i}$ and $p_{k}$, that is, 
$y\left(p_{i}\right)-y\left(p_{k}\right)$. Thus, the maximum width for top-anchored empty rectangular annuli is one among $O\left(n^{2}\right)$ values $\left\{y\left(p_{i}\right)-y\left(p_{k}\right) \mid 1 \leq i \leq k \leq n\right\}$.

The problem becomes even simpler if we fix $p_{i}$ on the top side of the outer rectangle, since the number of possible widths is reduced to $n$. An outlook of our algorithm that computes a maximum-width empty top-anchored rectangular annulus is as follows: (1) For each $p_{i} \in P$, find an empty annulus $A_{i}^{*}$ with $p_{i}$ lying on the top side of its outer rectangle whose width is the maximum among the set $\left\{y\left(p_{i}\right)-y\left(p_{k}\right) \mid i<k \leq n\right\}$ and then (2) output the one with maximum width among $A_{i}^{*}$ for all $i \in\{1, \ldots, n\}$. In order to compute $A_{i}^{*}$, we try all possible points $p_{j}$ that bound the bottom side of the outer rectangle.

In the following subsections, we first study the case where two points $p_{i}$ and $p_{j}$ on the top and bottom sides are fixed, and then move on to the case where only a point $p_{i}$ on the top side is fixed. More precisely, we discuss a decision algorithm when two points on the top and bottom sides are fixed, and exploit it as a sub-procedure to solve the other case.

\subsection{Decision when two points on top and bottom are fixed}

Suppose that we are given $p_{i}$ and $p_{j}$ with $1 \leq i+1<j \leq n$ or $j=\infty$, and we consider only empty rectangular anuuli whose outer rectangle contains $p_{i}$ and $p_{j}$ on its top and bottom sides, respectively.

Here, we consider the following decision problem.

Given: A positive real $w>0$
Task: Does there exist an empty rectangular annulus of width at least
$w$ whose outer rectangle contains $p_{i}$ and $p_{j}$ on its top and bottom
sides, respectively?

Let $D_{i j}(w)$ denote the outcome of the above decision problem.

Observation 5 If $D_{i j}(w)$ is TRUE, then $D_{i j}\left(w^{\prime}\right)$ is TRUE for any $w^{\prime} \leq w$. On the other hand, if $D_{i j}(w)$ is FALSE, then $D_{i j}\left(w^{\prime}\right)$ is FALSE for any $w^{\prime} \geq w$.

Let $P_{i j}:=\left\{p_{i+1}, \ldots, p_{j-1}\right\}$ for $i<j \leq n$, and $P_{i \infty}:=\left\{p_{i+1}, \ldots, p_{n}\right\}$. In the following, we show that the decision problem for a given width $w>0$ can be solved by a combination of certain operations on points $P_{i j}$, namely, the $y$-range $x$-neighbor query and the range maximumgap query. Each of the two operations is described as follows:

(i) The $y$-range $x$-neighbor query: Given three real numbers $\left(x, y_{1}, y_{2}\right)$, this operation is to find two points $q_{1}$ and $q_{2}$ in $P_{i j}$ such that $q_{1}$ is the rightmost one among points $P_{i j} \cap$ $[-\infty, x] \times\left[y_{1}, y_{2}\right]$ and $q_{2}$ is the leftmost one among points $P_{i j} \cap[x, \infty] \times\left[y_{1}, y_{2}\right]$. Either $q_{1}$ and $q_{2}$ may be undefined if there is no point of $P_{i, j}$ in the corresponding range. If $q_{1}$ is undefined, then we return $q_{1}$ as a point at infinity such that $x\left(q_{1}\right)=-\infty$ and $y\left(q_{1}\right)=y_{1}$; if $q_{2}$ is undefined, then we return $q_{2}$ such that $x\left(q_{2}\right)=\infty$ and $y\left(q_{2}\right)=y_{1}$.

(ii) The range maximum-gap query (in $x$-coordinates): Given two real numbers $\left(x_{1}, x_{2}\right)$, find the maximum gap in the set of real numbers $\left\{x(p) \mid x_{1} \leq x(p) \leq x_{2}, p \in P_{i j}\right\} \cup\left\{x_{1}, x_{2}\right\}$, where $x(p)$ denotes the $x$-coordinate of point $p$. The maximum gap in a set $X$ of real numbers is the maximum difference between two consecutive elements when $X$ is sorted. Notice that $x_{1}$ and $x_{2}$ are also included in the above set. Here, the output of the range maximum-gap query is to be the pair of values that define the maximum gap.

We describe our algorithm for the decision problem as Algorithm 1

Our decision algorithm, Algorithm 1, evaluates $D_{i j}(w)$ for a given $w$. As described in Algorithm 1 the decision is made by four calls of the $y$-range $x$-neighbor queries and the range 

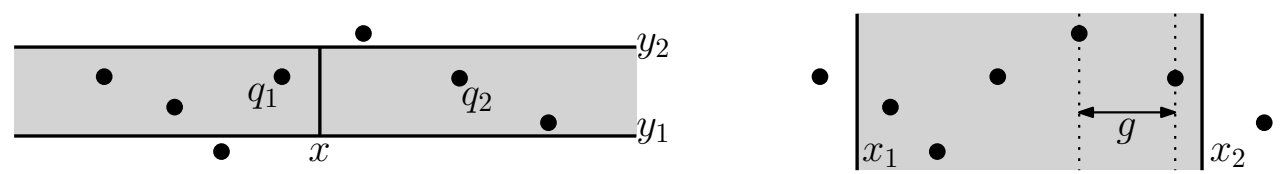

Figure 4: (left) the $y$-range $x$-neighbor query for $\left(x, y_{1}, y_{2}\right)$ and its answer $q_{1}$ and $q_{2}$, (right) the range maximum-gap query for $\left(x_{1}, x_{2}\right)$ and the maximum gap is $g$.

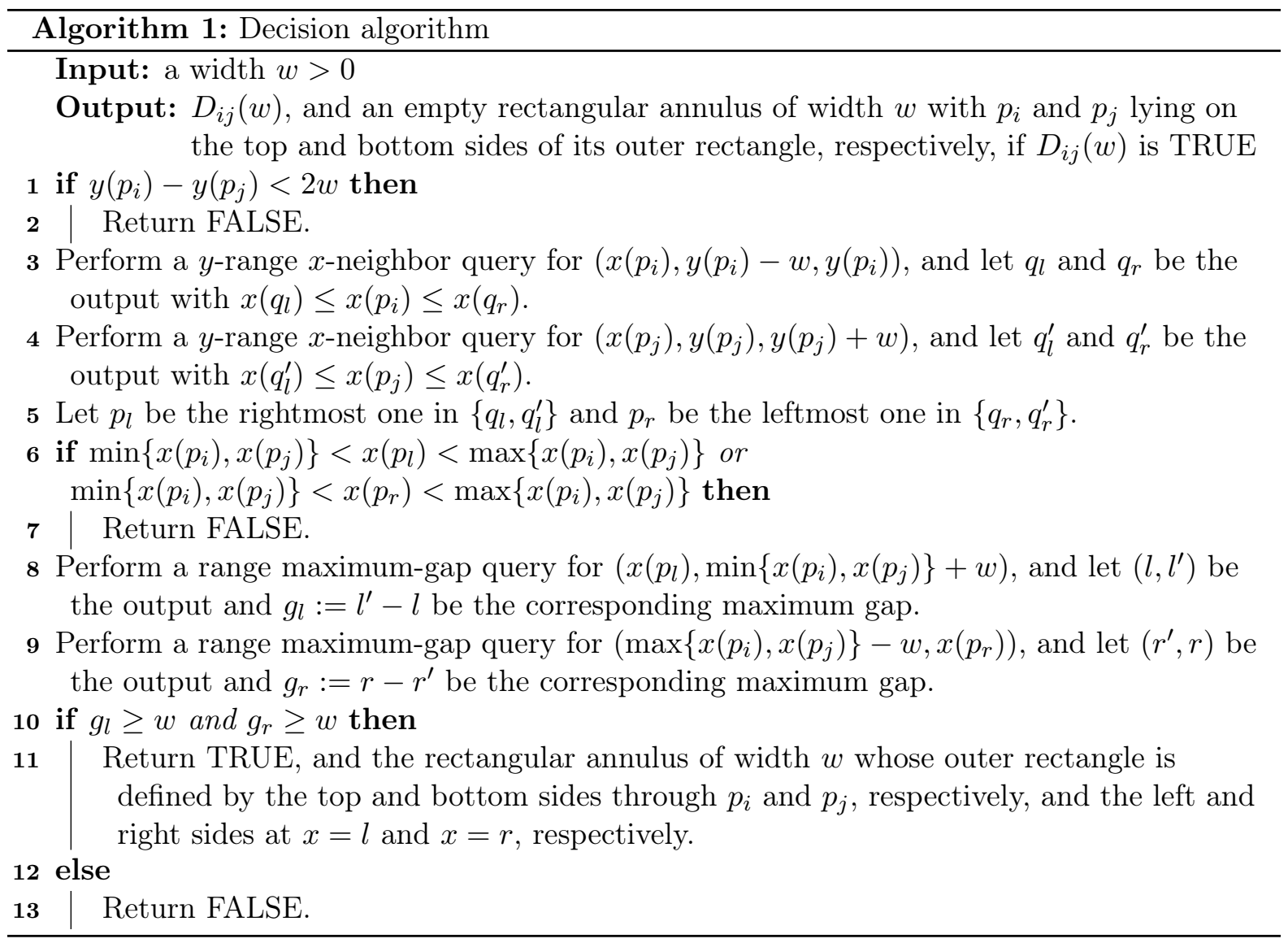

maximum-gap queries. Thus, its running time depends on how efficiently we can handle these queries. If the algorithm decides that $D_{i j}(w)$ is TRUE, then it also returns a corresponding rectangular annulus, that is, an empty uniform annulus of width $w$ with $p_{i}$ and $p_{j}$ on the top and bottom sides of the outer rectangle. This can be done by constructing its outer rectangle since its width $w$ is fixed.

In the following, we show the correctness of our decision algorithm.

Lemma 2 Algorithm 1 correctly computes $D_{i j}(w)$ for any given $w>0$ in time $O(T)$, where $T$ is an upper bound on time needed to perform a $y$-range $x$-neighbor query or a range maximum-gap query. Moreover, if $D_{i j}(w)$ is TRUE, then an empty rectangular annulus of width $w$ such that $p_{i}$ and $p_{j}$ lie on the top and bottom side of its outer rectangle can be found in the same time bound.

Proof. It is straightforward to see that the time complexity is $O(T)$, since it runs the $y$-range $x$-neighbor queries and the range maximum-gap queries at most four times. Hence, we focus on the correctness proof. Without loss of generality, we assume that $x\left(p_{i}\right) \leq x\left(p_{j}\right)$.

First, we show that if Algorithm 1 for input $w$ returns FALSE, then $D_{i j}(w)$ is FALSE. There 
are two cases where Algorithm 1 returns FALSE: Algorithm 1 returns FALSE either (1) at line 2 , (2) at line 7 or (3) at line 13. If $y\left(p_{i}\right)-y\left(p_{j}\right)<2 w$, then we cannot build a rectangular annulus of width $w$ with $p_{i}$ and $p_{j}$ on the top and bottom sides of its outer rectangle. Thus, if Algorithm 1 returns FALSE either at line 2, then this is clearly correct.

Suppose case (2), so Algorithm 1 returns FALSE at line 7. From lines 3 and 4, we have the following:

- $q_{1}$ is the rightmost point in $P_{i j} \cap\left(\left[-\infty, x\left(p_{i}\right)\right] \times\left[y\left(p_{i}\right)-w, y\left(p_{i}\right)\right]\right)$.

- $q_{r}$ is the leftmost point in $P_{i j} \cap\left(\left[x\left(p_{i}\right), \infty\right] \times\left[y\left(p_{i}\right)-w, y\left(p_{i}\right)\right]\right)$.

- $q_{1}^{\prime}$ is the rightmost point in $P_{i j} \cap\left(\left[-\infty, x\left(p_{j}\right)\right] \times\left[y\left(p_{j}\right), y\left(p_{i}\right)+w\right]\right)$.

- $q_{r}^{\prime}$ is the leftmost point in $P_{i j} \cap\left(\left[x\left(p_{j}\right), \infty\right] \times\left[y\left(p_{j}\right), y\left(p_{j}\right)+w\right]\right)$.

Also, by line $5, p_{l}$ is the rightmost one among $\left\{q_{l}, q_{l}^{\prime}\right\}$, and $p_{r}$ is the leftmost one among $\left\{q_{r}, q_{r}^{\prime}\right\}$. Then, in this case, we have

$$
x\left(p_{i}\right)<x\left(p_{l}\right)<x\left(p_{j}\right) \text { or } x\left(p_{i}\right)<x\left(p_{r}\right)<x\left(p_{j}\right),
$$

by the condition in line 6 . Assume the first condition holds, so $x\left(p_{i}\right)<x\left(p_{l}\right)<x\left(p_{j}\right)$. This implies that $p_{l}=q_{l}^{\prime}$. By our construction, we have $y\left(p_{l}\right)=y\left(q_{l}^{\prime}\right) \in\left[y\left(p_{j}\right), y\left(p_{j}\right)+w\right]$. Consider now any rectangular annulus $A$ of width $w$ whose outer rectangle $R_{\text {out }}$ contains $p_{i}$ and $p_{j}$ on its top and bottom sides, respectively, and observe that $A$ must contain the point $p_{l}$. Thus, such an annulus $A$ cannot be empty, so $D_{i j}(w)$ is FALSE. The other case where it holds that $x\left(p_{i}\right)<x\left(p_{r}\right)<x\left(p_{j}\right)$ can be handled analogously.

Now, suppose case (3), so Algorithm 1 returns FALSE at line 13. Then, by lines 8 and 9, so that we have

- $\left(l, l^{\prime}\right)$ defines the maximum gap $g_{l}=l^{\prime}-l$ in range $\left[x\left(p_{l}\right), x\left(p_{i}\right)+w\right]$.

- $\left(r^{\prime}, r\right)$ defines the maximum gap $g_{r}=r-r^{\prime}$ in range $\left[x\left(p_{j}\right)-w, x\left(p_{r}\right)\right]$.

In this case, we have

$$
g_{l}<w \quad \text { or } \quad g_{r}<w,
$$

by the condition in line 10. Assume that the first condition holds, so $g_{l}<w$. This means that there is no vertical strip of width at least $w$ among points in $P_{i j}$ in the $x$-range $\left[x\left(p_{l}\right), x\left(p_{i}\right)+w\right]$. Consider now any rectangular annulus $A$ of width $w$ whose outer rectangle $R_{\text {out }}$ contains $p_{i}$ and $p_{j}$ on its top and bottom sides, respectively. If the left side of $R_{\text {out }}$ is to the left of $p_{l}$, then $A$ contains $p_{l}$ by the construction; otherwise, any vertical strip of width $w$ between $\left[x\left(p_{l}\right), x\left(p_{i}\right)+w\right]$ contains a point in $P_{i j}$. Since the left side of $R_{o u t}$ cannot be to the right of $p_{i}$, this implies that no such annulus $A$ is empty in this case, so $D_{i j}(w)$ is indeed FALSE. The other case where it holds that $g_{r}$ can be handled analogously.

Next, we show that if Algorithm 1 for input $w$ returns TRUE, then $D_{i j}(w)$ is correctly TRUE. If Algorithm 1 for input $w$ returns TRUE, then we have the following:

$$
x\left(p_{l}\right) \leq x\left(p_{i}\right), x\left(p_{r}\right) \geq x\left(p_{r}\right), g_{l} \geq w, g_{r} \geq w,
$$

by the conditions in lines 6 and 10. Then, we can construct a rectangle $R_{\text {out }}$ such that its top and bottom sides go through $p_{i}$ and $p_{j}$, respectively, and the $x$-coordinates of its left and right sides are $l$ and $r$, respectively. Let $A$ be the rectangular annulus of width $w$ whose outer rectangle is $R_{\text {out }}$. Then, by our construction, $A$ is empty with respect to $P_{i j}$. Since $A$ is completely contained in the horizontal strip defined by the horizontal lines through $p_{i}$ and $p_{j}, A$ is also empty with 
respect to the whole set $P$. This shows the existence of an empty annulus $A$ of width $w$ such that the top and bottom sides of its outer rectangle contains $p_{i}$ and $p_{j}$, respectively. Therefore, we conclude that $D_{i j}(w)$ is TRUE. In this case, Algorithm 1 indeed returns the annuls $A$. This completes the proof.

Note that the two operations can be easily done in linear time. We now show how to perform them in logarithmic time with an aid of the following data structures.

- Let $\mathcal{D}$ be the data structure on $P$ described in Chazelle $[6]$ that supports a segment dragging query for vertical line segments dragged by two horizontal rays. A segment dragging query is given by a segment and a direction along two rays and is to find the first point in $P$ that is hit by the dragged segment. This structure can be constructed in $O(n \log n)$ time using $O(n)$ storage

- Let $\mathcal{X}_{i j}$ be a $1 \mathrm{D}$ range tree for the $x$-coordinates of points in $P_{i j}$ with an additional field $\operatorname{maxgap}(v)$ at each node $v$, where $\operatorname{maxgap}(v)$ denotes the maximum gap in the canonical subset of $v$. Note that $\operatorname{maxgap}(v)=0$ if the canonical subset of $v$ consists of only one element. The structure $\mathcal{X}_{i j}$ can be constructed using storage $O\left(\left|P_{i j}\right|\right)[8]$.

Now, suppose that we have already built these two structures $\mathcal{D}$ and $\mathcal{X}_{i j}$. Then, the two operations can be handled in $O(\log n)$ time as follows:

(i) For a $y$-range $x$-neighbor query for $\left(x, y_{1}, y_{2}\right)$, we perform two segment dragging queries on $\mathcal{D}$ for a vertical line segment with endpoints $\left(x, y_{1}\right)$ and $\left(x, y_{2}\right)$ to both the left and the right directions. These two queries result in the rightmost point $q_{1}$ in the range $[-\infty, x] \times\left[y_{1}, y_{2}\right]$ and the leftmost point $q_{2}$ in the range $[x, \infty] \times\left[y_{1}, y_{2}\right]$.

(ii) For a range maximum-gap query for $\left(x_{1}, x_{2}\right)$, we perform a $1 \mathrm{D}$ range search for the $x$-range $\left[x_{1}, x_{2}\right]$ on $\mathcal{X}_{i j}$ again to obtain a collection $C$ of $O(\log n)$ nodes. The maximum gap in the $x$-coordinates of the points in $P_{i j}$ in the range $\left[x_{1}, x_{2}\right]$ can be found by comparing $O(\log n)$ values: $\operatorname{maxgap}(v)$ for all $v \in C$ and every gap between two consecutive canonical subset.

Therefore, we conclude the following:

Lemma 3 Suppose that we already have two tree structures $\mathcal{D}$ and $\mathcal{X}_{i j}$. Then, Algorithm 1 correctly computes $D_{i j}(w)$ for any given $w>0$ in time $O(\log n)$.

\subsection{Optimization when only a point on top is fixed}

Next, we describe how to find a maximum-width empty top-anchored rectangular annulus such that $p_{i}$ lies on the top side of the outer rectangle.

Let $w_{i}^{*}$ be the width of $A_{i}^{*}$. Observe that $w_{i}^{*}$ lies in the set $W_{i}:=\left\{y\left(p_{i}\right)-y\left(p_{k}\right) \mid i \leq k \leq\right.$ $n$ \}. Instead of solving the optimization problem for each pair $\left(p_{i}, p_{j}\right)$, we can rather solve the optimization problem when only a point $p_{i}$ on top is fixed. Our algorithm that computes $A_{i}^{*}$ and its width $w_{i}^{*}$ is presented as in Algorithm 2.

Lemma 4 Algorithm 2 can be implemented in $O(n \log n)$ time and $O(n)$ space for a fixed $p_{i} \in P$. Also, it correctly computes $w_{i}^{*}$ and $A_{i}^{*}$.

Proof. In Algorithm 2, we maintain several variables:

- $A$ is the currently best empty top-anchored annulus with $p_{i}$ on the top side of its outer rectangle.

- $w$ is the width of $A$.

- $k$ is the index such that the next larger width $y\left(p_{i}\right)-y\left(p_{k}\right)$ is currently being tried. It always holds that $w=y\left(p_{i}\right)-y\left(p_{k-1}\right)$. 


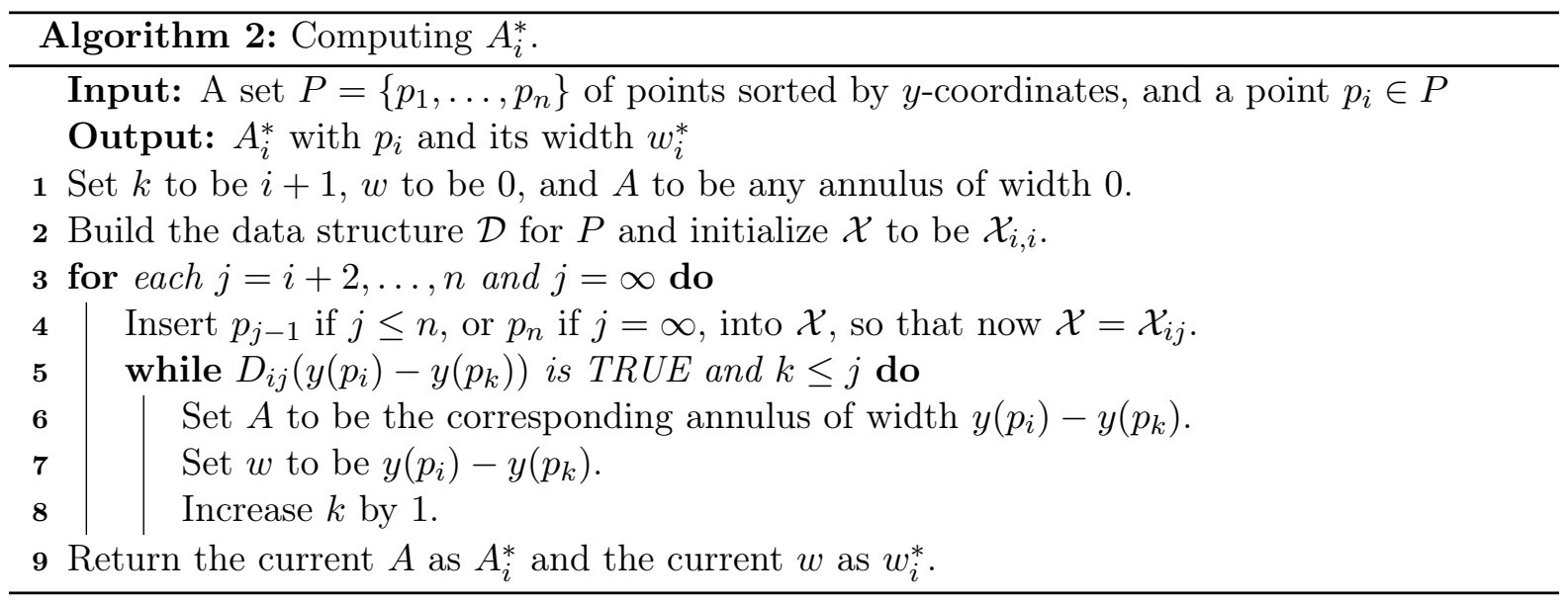

- $\mathcal{X}$ is the $1 \mathrm{D}$ range tree with additional field $\operatorname{maxgap}(v)$ at each node defined as above on the $x$-coordinates of the points in $P_{i j}$, that is, $\mathcal{X}=\mathcal{X}_{i j}$ in the main loop.

After initializing these variables properly in lines 1-2, the main loop runs for $j=i+2, \ldots, n$. Let $w_{i j}$ be defined as above. For each $j$, we keep the best annulus we have seen so far as $A$, not computing the exact value of $w_{i j}$. That is, we keep

$$
w=\max \left\{0, w_{i(i+2)}, w_{i(i+3)}, \ldots, w_{i j}\right\}
$$

as the loop invariant for each $j$. In this way, after the main loop has finished at $j=\infty$, we have

$$
w=\max _{j=i+2, \ldots, n, \infty} w_{i j}=w_{i}^{*}
$$

and the corresponding annulus of width $w=w_{i}^{*}$ is stored in variable $A$.

Hence, the correctness of Algorithm 2 is guaranteed. The time complexity is bounded by $O(n \log n)$ as follows.

Lines 1-2 in Algorithm 2 takes $O(n \log n)$ time since $\mathcal{D}$ can be built in $O(n \log n)$ time 6 and $\mathcal{X}_{i i}$ is initialized in $O(1)$ time as $P_{i, i}=\emptyset$. In line 4 , a point $p_{j-1}$ or $p_{n}$ is inserted into two tree data structures $\mathcal{T}$ and $\mathcal{X}$. The structures $\mathcal{X}$ is known to support an insertion in logarithmic time [8]. The additional information $\operatorname{maxgap}(v)$ at each node $v$ of $\mathcal{X}$ can be correctly updated in the bottom-up fashion through the path to the root from the newly inserted node. Hence, line 4 can be implemented in $O(\log n)$ time. In line 5, we call the decision algorithm, Algorithm 1 . and one execution of line 5 takes $O(\log n)$ time by Lemma 3 , since the necessary data structures $\mathcal{D}$ and $\mathcal{X}=\mathcal{X}_{i j}$ are provided. Lines 6-8 takes only $O(1)$ time.

To conclude the total running time, observe that line 4 is executed at most $n-3$ times. The "while" loop in line 5 is executed at most $2(n-3)$ times: The number of times when the while-condition is false is bounded by $n-3$, while the number of times when the while-condition is true is also bounded by $n-3$ since $k$ increases by one whenever this is the case and $k$ cannot be more than $n$.

Consequently, the time complexity of Algorithm 2 is bounded by $O(n \log n)$. Since the segment dragging query structure $\mathcal{D}$ and the $1 \mathrm{D}$ range tree $\mathcal{X}$ use $O(n)$ space [6,8], the space usage of Algorithm 2 is bounded by $O(n)$.

\subsection{Putting it all together}

We are now ready to describe the overall algorithm to solve the MaxERA problem. Under the assumption that there exists a maximum-width empty rectangular annulus $A^{*}$ that satisfies the 
condition of Observation 3 and is top-anchored, we excute Algorithm 2 for each $i=1, \ldots n-1$ and choose the one with the maximum width as $A^{*}$. Its correctness is guaranteed by Lemma 4. The other three cases where there is a maximum-width empty rectangular annulus that satisfies the condition of Observation 3 and is either bottom-anchored, left-anchored, or right-anchored, can be handled in a symmetric way. Thus, the overall algorithm runs for the four cases and outputs one with the maximum width.

Theorem 3 Given a set $P$ of $n$ points in the plane, a maximum-width rectangular annulus that is empty with respect to $P$ can be computed in $O\left(n^{2} \log n\right)$ time and $O(n)$ space.

Proof. The correctness follows from the above discussion and Observation 4.

For the time complexity, we mainly call Algorithm $2 O(n)$ times. Thus, it takes $O\left(n^{2} \log n\right)$ time by Lemma 4 . The space usage can be bounded by $O(n)$ again by Lemma 4 .

\section{Concluding Remarks}

In this paper, we addressed the problem of computing a maximum-width empty square or rectangular annulus that avoids a given set $P$ of $n$ points in the plane, and presented two efficient algorithms. Our algorithms run in $O\left(n^{3}\right)$ and $O\left(n^{2} \log n\right)$ time, respectively, for a square and rectangular annulus. Note that our algorithms are first nontrivial algorithms that solve the problems, and considered to be efficient compared to the currently best algorithm for the circular counterpart, which runs in $O\left(n^{3} \log n\right)$ time [10].

There are two obvious open questions. One asks an improved algorithm with less running time, while the other asks a lower bound of the empty annulus problem. The circular, square, and rectangular versions of the problem are now known to be solved in $O\left(n^{3} \log n\right), O\left(n^{3}\right)$, and $O\left(n^{2} \log n\right)$ time, respectively. At this moment, it seems difficult to improve each of these upper bounds. On the other hand, no nontrivial lower bound, other than $\Omega(n)$, is known for these problems. This simply means that nobody fully understand the intrinsic complexity of this type of problems for now. It will be very interesting hence if one improves one of these algorithms or proves a nontrivial lower bound for the problem.

\section{References}

[1] M. Abellanas, F.Hurtado, C.Icking, L.Ma, B.Palop, and P.Ramos. Best fitting rectangles. In Proc. Euro. Workshop Comput. Geom. (EuroCG'03), 2003.

[2] P. K. Agarwal and M. Sharir. Efficient algorithms for geometric optimization. ACM Comput. Survey, 30(4):412-458, 1998.

[3] P. K. Agarwal, M. Sharir, and S. Toledo. Applications of parametric searching in geometric optimization. J. Algo., 17(3):292-318, 1994.

[4] A. Aggarwal and S. Suri. Fast algorithms for computing the largest empty rectangle. In Proc. the Third Annu. Sympos. Comput. Geom. (SoCG'87), pages 278-290, 1987.

[5] S. W. Bae. Computing a minimum-width square annulus in arbitrary orientation. Theoret. Comput. Sci., 718:2-13, 2018.

[6] B. Chazelle. An algorithm for segment-dragging and its implementation. Algorithmica, 3(1):205-221, 1988. 
[7] S.-W. Cheng. Widest empty L-shaped corridor. Inform. Proc. Lett., 58(6):277 - 283, 1996.

[8] M. de Berg, O. Cheong, M. van Kreveld, and M. Overmars. Computational Geometry: Algorithms and Applications. Springer-Verlag TELOS, Santa Clara, CA, USA, 3rd ed. edition, 2008.

[9] J. Díz-Báñez, M. López, and J. Sellarès. On finding a widest empty 1-corner corridor. Inform. Proc. Lett., 98(5):199 - 205, 2006.

[10] J. M. Díz-Báñez, F. Hurtado, H. Meijer, D. Rappaport, and J. A. Sellarès. The largest empty annulus problem. Int. J. Comput. Geom. Appl., 13(4):317-325, 2003.

[11] H. Ebara, N. Fukuyama, H. Nakano, and Y. Nakanishi. Roundness algorithms using the Voronoi diagrams. In Abstract: 1st Canadian Conf. Comput. Geom. (CCCG'89), page 41, 1989.

[12] O. N. Gluchshenko, H. W. Hamacher, and A. Tamir. An optimal $O(n \log n)$ algorithm for finding an enclosing planar rectilinear annulus of minimum width. Oper. Res. Lett., $37(3): 168-170,2009$.

[13] J. Hershberger. Finding the upper envelope of $n$ line segments in $O(n \operatorname{logn})$ time. Inform. Proc. Lett., 33(4):169 - 174, 1989.

[14] M. Houle and A. Maciel. Finding the widest empty corridor through a set of points. In G. Toussaint, editor, Snapshots of computational and discrete geometry, pages 201-213. Dept. Computer Science, McGill University, 1988.

[15] R. Janardan and F. P. Preparata. Widest-corridor problems. Nordic J. Comput., 1:231 $245,1994$.

[16] P. R. S. Mahapatra. Largest empty axis-parallel rectangular annulus. Journal of Emerging Trends in Computing and Information Sciences, 3(6), 2012.

[17] J. Mukherjee, P. R. S. Mahapatra, A. Karmakar, and S. Das. Minimum-width rectangular annulus. Theoret. Comput. Sci., 508:74-80, 2013.

[18] F. P. Preparata and M. I. Shamos. Computational Geometry: an Introduction. Springer, 1990.

[19] U. Roy and X. Zhang. Establishment of a pair of concentric circles with the minimum radial separation for assessing roundness error. Computer-Aided Design, 24(3):161-168, 1992.

[20] G. T. Toussaint. Computing largest empty circles with location constraints. Int. J. Comput. Info. Sci., 12(5):347-358, 1983.

[21] G. T. Toussaint. Solving geometric problems with the rotating calipers. In Proc. the IEEE MELECON 83, pages 1-4, 1983.

[22] A. D. Wainstein. A non-monotonous placement problem in the plane. In Abstract: 9th AllUnion Symposium USSR Software Systems for Solving Optimal Planning Problems, pages 70-71, 1986. 